\title{
TIGHT CONTACT STRUCTURES WITH NO SYMPLECTIC FILLINGS
}

\author{
JOHN B. ETNYRE AND KO HONDA
}

\begin{abstract}
We exhibit tight contact structures on 3-manifolds that do not admit any symplectic fillings.
\end{abstract}

\section{INTRODUCTION}

In the early 1980's, D. Bennequin [2] proved the existence of exotic contact structures on $\mathbf{R}^{3}$. These were obtained from the standard contact structure on $\mathbf{R}^{3}$ given by the 1 -form $\alpha=d z-y d x$, by performing modifications called Lutz twists. The key distinguishing feature was that the exotic contact structures contained overtwisted disks, i.e., disks $D$ which are everywhere tangent to the 2-plane field distribution along $\partial D$. On the other hand, using an ingenious argument which used braid foliations, Bennequin succeeded in proving that the standard contact structure contained no overtwisted disks. In a strange twist of fate, the exotic contact structures eventually turned out not to be so exotic, when Eliashberg [6] gave a complete classification of contact structures which contain overtwisted disks (called overtwisted contact structures) in terms of homotopy theory.

With the advent of Gromov's theory of holomorphic curves [20], it became easier to determine when a contact structure on a 3-manifold is tight, i.e., contains no overtwisted disks [8]. Loosely speaking, a contact structure is symplectically fillable if it is the boundary of a symplectic 4-manifold. Gromov and Eliashberg showed that a symplectically fillable structure was necessarily tight. In fact, until the mid-1990's, all known tight contact structures were shown to be tight using symplectic fillings. This included two rich sources of tight contact structures - perturbations of taut foliations as in [12] and Legendrian surgery as in [7] and [33]. This promoted Eliashberg and others to ask whether tight contact structures are the same as symplectically fillable contact structures. Subsequently, gluing techniques were developed by Colin [3, 4] and Makar-Limanov [29], and strengthened in [23]. Largely due to the improvements in gluing techniques, tight contact

Date: First version: October 4, 2000. This version: October 17, 2000.

1991 Mathematics Subject Classification. Primary 57M50; Secondary 53C15.

Key words and phrases. tight contact structure, symplectically fillable, Legendrian surgery.

JE supported in part by NSF Grant \# DMS-9705949. KH supported in part by NSF Grant \# DMS-0072853 and the American Institute of Mathematics. 
structures could now be constructed without resorting to symplectic filling techniques. The main result of this paper shows that the symplectically fillable contact structures form a proper subset of tight contact structures.

Theorem 1.1. Let $M_{1}$ (resp. $M_{2}$ ) be the Seifert fibered space over $S^{2}$ with Seifert invariants $\left(-\frac{1}{2}, \frac{1}{4}, \frac{1}{4}\right)$ (resp. $\left(-\frac{2}{3}, \frac{1}{3}, \frac{1}{3}\right)$ ). Then $M_{1}$ admits one tight contact structure and $M_{2}$ admits two nonisotopic tight contact structures that are not weakly symplectically semi-fillable.

In this paper we will provide a complete proof for $M=M_{1}$; the proof for $M=M_{2}$ is similar, and we will briefly discuss the necessary modifications at the end of Section 3 .

Remark on notation. A Seifert fibered space over a closed oriented surface $\Sigma$ with $n$ singular fibers is often denoted by $\left(g ;(1, e),\left(\alpha_{1}, \beta_{1}\right), \cdots,\left(\alpha_{n}, \beta_{n}\right)\right)$, or by $\left(g ; e, \frac{\beta_{1}}{\alpha_{1}}, \cdots, \frac{\beta_{n}}{\alpha_{n}}\right)$, where $g$ is the genus of the base $\Sigma, e \in \mathbf{Z}$ is the Euler number, and $\alpha_{i}, \beta_{i} \in \mathbf{Z}^{+}$are relatively prime. In this notation, $\left(-\frac{1}{2}, \frac{1}{4}, \frac{1}{4}\right)$ would correspond to $\left(0 ;-1, \frac{1}{2}, \frac{1}{4}, \frac{1}{4}\right)$ and $\left(-\frac{2}{3}, \frac{1}{3}, \frac{1}{3}\right)$ to $\left(0 ;-1, \frac{1}{3}, \frac{1}{3}, \frac{1}{3}\right)$.

\section{BACKGROUND AND PRELIMINARY NOTIONS}

We briefly review the basic notions in Section 2.1 and proceed to a discussion of symplectic fillings in Section 2.2. There we introduce the various types of symplectic fillings and discuss the work of Lisca concerning the nonexistence of fillable structures on certain manifolds. Finally, in Section 2.3, we discuss the contact surgery technique which is usually called Legendrian surgery.

Convex surface theory will be our main tool throughout this paper. Originally developed by Giroux in [16], there have been many recent papers discussing convex surfaces. All the facts relevant to this paper concerning convex surfaces may be found in [21, 15] (see also [24, 22]), and we assume the reader is familiar with the terminology in these papers.

2.1. Contact structures and Legendrian knots. In this section we review a few basic notions of contact topology in dimension three. This is more to establish terminology than to introduce the readers to these ideas. Readers unfamiliar with these ideas should see [1, 9].

An oriented 2-plane field distribution $\xi$ on an oriented 3-manifold $M$ is called a positive contact structure if $\xi=\operatorname{ker} \alpha$ for some global 1-form $\alpha$ satisfying $\alpha \wedge d \alpha>0$. The 1 -form $\alpha$ is called the contact form for $\xi$. In this paper we will always assume that our ambient manifold $M$ is oriented and the contact structure $\xi$ is positive and oriented. If $\Sigma$ is a surface in a contact manifold $(M, \xi)$, then $\Sigma$ has a singular foliation $\Sigma_{\xi}$, called the characteristic foliation, given by integrating the singular line filed $T_{x} \Sigma \cap \xi_{x}$. It is important to remember that the characteristic foliation on a surface determines the germ of the contact structure along the surface. A contact structure $\xi$ is called overtwisted if there is an embedded disk $D$ which is everywhere 
tangent to $\xi$ along $\partial D$. A contact structure is tight if it is not overtwisted. For a complete classification of overtwisted contact structures see [6]. A tight contact structure $\xi$ on $M$ is called universally tight if it remains tight when pulled back to the universal cover, and is called virtually overtwisted if it becomes overtwisted when pulled back to some finite cover. It is an interesting problem to determine whether every tight contact structure is either universally tight or virtually overtwisted.

A knot $K$ embedded in a contact manifold $(M, \xi)$ is called Legendrian if it is everywhere tangent to $\xi$. A choice of nonzero section of $\xi$ transverse to $K$ gives a framing of the normal bundle of $K$, usually called the contact framing. If $\mathcal{F}$ is some preassigned framing of $K$, then we associate an integer called the twisting number of $\xi$ along $K$ relative to $\mathcal{F}$, which is the difference in twisting between the contact framing and $\mathcal{F}$, and denote it $t(K, \mathcal{F}$ ) (or just $t(K)$ if the framing $\mathcal{F}$ is understood). If $K$ is a null-homologous knot and $\mathcal{F}$ is given by a Seifert surface for $K$, then $t(K)$ is called the ThurstonBennequin invariant of $K$ and is usually denoted $t b(K)$.

A closed surface or a properly embedded compact surface $\Sigma$ with Legendrian boundary is called convex if there exists a contact vector field everywhere transverse to $\Sigma$. To a convex surface $\Sigma$ we associate an isotopy class of multicurves called the dividing set $\Gamma_{\Sigma}$ (or simply $\Gamma$ ). If $\Sigma$ is closed, then components of $\Gamma_{\Sigma}$ are closed curves, and if $\Sigma$ has boundary, there may also be properly embedded arcs. The number of components of $\Gamma_{\Sigma}$ is written as $\# \Gamma_{\Sigma}$. The Flexibility Theorem of Giroux [16] states that $\Gamma_{\Sigma}$ (not the precise characteristic foliation) encodes all the contact-topological information in a small neighborhood of $\Sigma$. The complement of the dividing set is the union of two subsets $\Sigma \backslash \Gamma_{\Sigma}=\Sigma_{+}-\Sigma_{-}$. Here $\Sigma_{+}$is the subsurface where the orientation of $\Sigma$ and the normal orientation of $\xi$ coincide, and $\Sigma_{-}$is the subsurface where they are opposite. Therefore, we can refer to positive and negative components of $\Sigma \backslash \Gamma_{\Sigma}$.

2.2. Symplectic fillings. The easiest way to prove a contact structure is tight is to show it 'bounds' a symplectic 4-manifold. There are several notions of 'symplectic filling', and we assemble the various notions here for the convenience of the reader. (For more details, see the survey paper [13].)

A symplectic manifold $(X, \omega)$ is said to have $\omega$-convex boundary if there is a vector field $v$ defined in the neighborhood of $\partial X$ that points transversely out of $X$ and for which $\mathcal{L}_{v} \omega=\omega$, where $\mathcal{L}$ denotes the Lie derivative. One may easily check that $\alpha=\left.\left(\iota_{v} \omega\right)\right|_{\partial X}$ is a contact form on $\partial X$. A symplectic manifold $(X, \omega)$ is said to have weakly convex boundary if $\partial X$ admits a contact structure $\xi$ such that $\left.\omega\right|_{\xi}>0$ (and the orientations induced on $\partial X$ by $X$ and $\xi$ agree). A contact structure $\xi$ on $M$ is:

1. Holomorphically fillable if $(M, \xi)$ is the $\omega$-convex boundary of some Stein manifold $(X, \omega)$.

2. Strongly symplectically fillable if $(M, \xi)$ is the $\omega$-convex boundary of some symplectic manifold $(X, \omega)$. 
3. Weakly symplectically fillable if $(M, \xi)$ is the weakly convex boundary of some symplectic manifold $(X, \omega)$.

4. Weakly symplectically semi-fillable if $(M, \xi)$ is one component of the weakly convex boundary of some symplectic manifold $(X, \omega)$.

Theorem 2.1 (Gromov-Eliashberg). Let $(M, \xi)$ be a contact 3-manifold which satisfies any of the above conditions for fillability. Then $\xi$ is tight.

The following diagram indicates the hierarchy of contact structures.

\begin{tabular}{|ccc|}
\hline Tight & & \\
$\cup$ X & & \\
Weakly symplectically semi-fillable & $\supsetneqq$ & Strongly symplectically semi-fillable \\
Weakly symplectically fillable & $\supsetneqq$ & Strongly symplectically fillable \\
& & Holomorphically fillable \\
& & \\
\end{tabular}

The proper inclusion of the set of weakly symplectically semi-fillable contact structures into the set of tight contact structures is the content of Theorem 1.1. The proper inclusion of the set of strongly fillable structures into the set of weakly fillable structures is already seen on $T^{3}$ (due to Eliashberg [10]). This result was recently extended by Fan Ding to $T^{2}$-bundles over $S^{1}$. For all other inclusions in the diagram it is not known whether the inclusions are strict.

We briefly discuss when the various notions of fillability become the same. If $H^{2}(M ; \mathbf{Q})=0$, a weak symplectic filling can be modified into a strong symplectic filling [31]. Using work of Kronheimer and Mrowka [26] and Seiberg-Witten theory, Lisca 27] showed that if $M$ has a positive scalar curvature metric, then a semi-filling is automatically a one-component filling (there is also a related, but weaker, result in Ohta-Ono [31]).

Lisca [27] went further to show (among other things) that:

Theorem 2.2 (Lisca [27]). Let $M$ be a Seifert fibered space over $S^{2}$ with Seifert invariants $\left(-\frac{1}{2}, \frac{1}{3}, \frac{1}{4}\right)$ or $\left(-\frac{1}{2}, \frac{1}{3}, \frac{1}{3}\right)$. The manifold $M$ does not carry a weakly symplectically semi-fillable contact structure.

In Lisca's paper the Seifert fibered space with invariants $\left(-\frac{1}{2}, \frac{1}{3}, \frac{1}{4}\right)$ is described as the boundary $M$ of the 4-manifold obtained by plumbing disk bundles over $S^{2}$ according to the positive $E_{7}$ diagram (left-hand side of Figure 11). It is an easy exercise in Kirby calculus [19] to show that $M$ is orientation-preserving diffeomorphic to the manifold shown on the righthand side of Figure 1, which is a presentation for a Seifert fibered space. Similarly, the Seifert fibered space with invariants $\left(-\frac{1}{2}, \frac{1}{3}, \frac{1}{3}\right)$ corresponds to the positive $E_{6}$ diagram. 

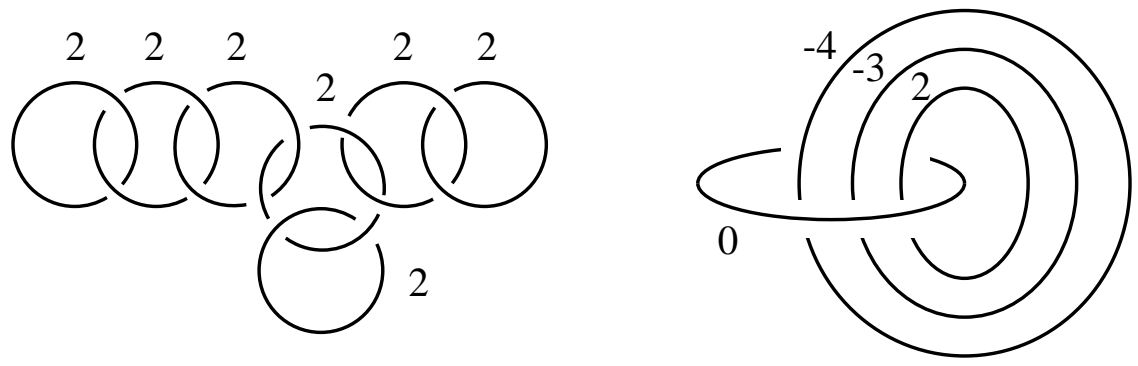

Figure 1. The plumbed disk bundles (left) and the Seifert fibered space $M$ (right).

2.3. Legendrian surgeries. Let us now describe a contact surgery techinque called Legendrian surgery. We first give a description on the 3manifold level. Given a Legendrian knot $L$ in any contact 3 -manifold $(M, \xi)$, a Legendrian surgery on $L$ yields the contact manifold $\left(M^{\prime}, \xi^{\prime}\right)$, where $M^{\prime}$ is obtained from $M$ by $t(L)-1$ Dehn surgery on $L$ and $\xi^{\prime}$ is obtained from $\xi$ as follows: Let $N$ be a standard convex neighborhood of $L$. Choose a framing on $N$ so that $t(L)=0$. This choice of framing allows us to make an oriented identification $-\partial(M \backslash N) \simeq \mathbf{R}^{2} / \mathbf{Z}^{2}$, where $(1,0)^{T}$ is the meridian of $N$ and $(0,1)^{T}$ is the longitude of $N$ corresponding to the framing. Now take an identical copy $N^{\prime}$ of $N$ (with the same framing), and make an oriented identification $\partial N^{\prime} \simeq \mathbf{R}^{2} / \mathbf{Z}^{2}$, where $(1,0)^{T}$ is the meridian and $(0,1)^{T}$ is the longitude. Then let $M^{\prime}=(M \backslash N) \cup_{\psi} N^{\prime}$ where $\psi: \partial N^{\prime} \stackrel{\sim}{\rightarrow}-\partial(M \backslash N)$ is represented by the matrix $\left(\begin{array}{cc}1 & 0 \\ -1 & 1\end{array}\right) \in S L(2, \mathbf{Z})$. Since $\psi\left(\Gamma_{\partial N^{\prime}}\right)$ and $\Gamma_{\partial(M \backslash N)}$ are isotopic, we may use Giroux's Flexibility Theorem to arrange the characteristic foliation on $\partial N^{\prime}$ and isotop $\psi$ so $\psi_{*}\left(\left.\xi\right|_{N^{\prime}}\right)=\left.\xi\right|_{M \backslash N}$. Hence we may glue the contact structures on $N^{\prime}$ and $M \backslash N$.

Theorem 2.3. Legendrian surgery is category-preserving for each category in the diagram of inclusions above, with the possible exception of the category of tight contact structures.

Eliashberg [0] proved that Legendrian surgery is category-preserving for holomorphically fillable contact structures. Weinstein [33] proved Theorem 2.3 for strongly symplectically fillable contact structures.

On the 4-manifold level, Legendrian surgery is described as follows: Let $(X, \omega)$ be a symplectic 4-manifold with $\omega$-convex boundary and $L$ a Legendrian knot in the induced contact structure on $\partial X$. If $X^{\prime}$ is obtained from $X$ by adding a 2 handle to $\partial X$ along $L$ with framing $t(L)-1$, then $\omega$ extends to a symplectic form $\omega^{\prime}$ on $X^{\prime}$ so that $\partial X^{\prime}$ is $\omega^{\prime}$-convex. For many interesting applications of Theorem 2.3, we refer the reader to Gompf [18].

The case of Theorem 2.3 known to a few experts but surprisingly absent in the literature is for the category of weakly fillable contact structures. The proof of Theorem 2.3 in the strongly fillable case relies only on the 
symplectic structure on $X$ in a neighborhood of $L$. Hence Theorem 2.3 in the weakly fillable case follows from:

Lemma 2.4. Let $(M, \xi)$ be a weakly symplectically fillable contact 3-manifold, $(X, \omega)$ one of its weak fillings, and $L$ a Legendrian knot in $(M, \xi)$. There is an arbitrarily small perturbation of $\xi$ in a neighborhood $N$ of $L$ so that $N$ is strongly convex. By this we mean there is a vector field $v$ defined on $X$ near $N$ so that $v$ points transversely out of $X, \mathcal{L}_{v} \omega=\omega$ and $\left.\xi\right|_{N}=\left.\operatorname{ker}\left(\iota_{v} \omega\right)\right|_{N}$.

Proof. Let $\left(M^{\prime}, \xi^{\prime}\right)$ be any strongly fillable contact 3-manifold, $\left(X^{\prime}, \omega^{\prime}\right)$ one of its strong fillings and $L^{\prime}$ a Legendrian knot in $\left(M^{\prime}, \xi^{\prime}\right)$. It is not hard to find a neighborhood $N$ of $L$ in $M$ and $N^{\prime}$ of $L^{\prime}$ in $M^{\prime}$ and a diffeomorphism $f: N \rightarrow N^{\prime}$ such that $f(L)=L^{\prime}, f^{*} \xi^{\prime}=\xi$ along $L$, and $f^{*}\left(\left.\omega^{\prime}\right|_{N^{\prime}}\right)=\left.\omega\right|_{N}$. One may then use standard arguments (see Exercise 3.35 in [30]) to extend $f$ to a symplectomorphism $(U, \omega) \stackrel{\sim}{\rightarrow}\left(U^{\prime}, \omega^{\prime}\right)$, where $U \subset X$ is a neighborhood of $N$ and $U^{\prime} \subset X^{\prime}$ is a neighborhood of $N^{\prime}$. Finally note that the contact planes $f^{*} \xi^{\prime}$ and $\xi$ agree on $L$ and are close together near $L$. By a small perturbation of $\xi$ near $L$ (small enough to keep $\xi$ contact so we may use Gray's Theorem), we may therefore assume $f^{*} \xi^{\prime}=\xi$ near $L$. Hence, if $v$ is the expanding vector field for $\omega^{\prime}$, then $f_{*}^{-1}(v)$ will be the desired vector field for $\omega$.

We now comment on Theorem 2.3 for the category of tight contact structures. In [23], it was shown that there exists a tight contact structure on a handlebody of genus 4 and a Legendrian surgery yielding an overtwisted contact structure. It is currently not known whether Legendrian surgery preserves tightness for closed 3-manifolds.

\section{The proof of the main Result for the Seifert fibered Space WITH INVARIANTS $\left(-\frac{1}{2}, \frac{1}{4}, \frac{1}{4}\right)$}

3.1. Seifert fibered spaces. Let $M$ be a Seifert fibered space over $S^{2}$ with three singular fibers $F_{1}, F_{2}, F_{3}$ and Seifert invariants $\left(\frac{\beta_{1}}{\alpha_{1}}, \frac{\beta_{2}}{\alpha_{2}}, \frac{\beta_{3}}{\alpha_{3}}\right)$. We describe $M$ explicitly as follows: Let $V_{i}, i=1,2,3$, be a tubular neighborhood of the singular fiber $F_{i}$. We identify $V_{i} \simeq D^{2} \times S^{1}$ and $\partial V_{i} \simeq \mathbf{R}^{2} / \mathbf{Z}^{2}$ by choosing $(1,0)^{T}$ as the meridional direction, and $(0,1)^{T}$ as the longitudinal direction given by $\{p t\} \times S^{1}$. We also identify $M \backslash\left(\cup_{i} V_{i}\right)$ with $\Sigma_{0} \times S^{1}$, where $\Sigma_{0}$ is a sphere with three punctures, and further identify $-\partial\left(M \backslash V_{i}\right)=\mathbf{R}^{2} / \mathbf{Z}^{2}$, by letting $(0,1)^{T}$ be the direction of an $S^{1}$-fiber, and $(1,0)^{T}$ be the direction given by $\partial\left(M \backslash V_{i}\right) \cap\left(\Sigma_{0} \times\{p t\}\right)$. With these identifications we may reconstruct $M$ from $\left(\Sigma_{0} \times S^{1}\right) \cup\left(\cup_{i=1}^{3} V_{i}\right)$ by gluing

$$
A_{i}: \partial V_{i} \stackrel{\sim}{\rightarrow}-\partial\left(M \backslash V_{i}\right), \quad A_{i}=\left(\begin{array}{cc}
\alpha_{i} & \gamma_{i} \\
-\beta_{i} & \delta_{i}
\end{array}\right) \in S L(2, \mathbf{Z}) .
$$

Note we have some freedom in choosing our matrices $A_{i}$ above. For example, in choosing $A_{i}$ we can alter $\gamma_{i}, \delta_{i}$ by altering our choice of framing for $V_{i}$, which will result in post-multiplying a given $A_{i}$ by $\left(\begin{array}{cc}1 & m \\ 0 & 1\end{array}\right)$. 
The twisting number of a Legendrian knot isotopic to a regular (i.e., nonsingular) fiber of the Seifert fibration will be measured using the framing from the product structure $\Sigma_{0} \times S^{1}$. (Whenever we say isotopy we will mean a smooth isotopy, as opposed to a contact isotopy.) On the other hand, a Legendrian knot isotopic to a singular fiber in a Seifert fibration will be measured with respect to the framing on $V_{i}$ chosen in the description for $M$.

Let us now specialize to the case where $M$ is given by Seifert invariants $\left(-\frac{1}{2}, \frac{1}{4}, \frac{1}{4}\right)$. We make the following choices:

$$
A_{1}=\left(\begin{array}{cc}
2 & -1 \\
1 & 0
\end{array}\right), \quad A_{2}=A_{3}=\left(\begin{array}{cc}
4 & 1 \\
-1 & 0
\end{array}\right) .
$$

From now on $M$ will refer to this particular Seifert fibered space.

3.2. Description as a torus bundle. To define the contact structure $\xi$ in Theorem 1.1 and prove tightness, we need a description of $M$ as a torus bundle over $S^{1}$. Recall a torus bundle over $S^{1}$ can be described as

$$
T^{2} \times[0,1] / \sim
$$

where $(A x, 0) \sim(x, 1)$ and $A$ is in $S L(2, \mathbf{Z})$. The matrix $A$ is called the monodromy of the torus bundle.

Lemma 3.1. The manifold $M$ is a torus bundle over $S^{1}$ with monodromy $A=\left(\begin{array}{cc}0 & 1 \\ -1 & 0\end{array}\right)$.

Proof. The map $A$ has order four with two fixed points and two points of order two (which are interchanged under $A$ ). One may thus conclude that the torus bundle is a Seifert fibered space over $S^{2}$ with Seifert invariants $\left( \pm \frac{1}{2}, \pm \frac{1}{4}, \pm \frac{1}{4}\right)$. To determine the sign of invariants, let $D \subset T^{2}$ be a small disk about one of the fixed points with $A(D)=D$, and consider $S=D \times[0,1] / \sim$. If $x \in \partial D$, then a regular fiber in the Seifert fibered structure will be given by $\left(\left\{A^{i}(x) \mid i=0,1,2,3\right\} \times[0,1]\right) / \sim$. One may pick a product structure on $S$ so that a regular fiber will be a $(-1,4)$-curve on $\partial S$. From this we see that the gluing matrix $A_{i}$ associated to this singular fiber is $\left(\begin{array}{cc}4 & 1 \\ -1 & 0\end{array}\right)$. Thus two of the Seifert invariants are $\frac{1}{4}$. Similarly, one may check that the third invariant is $-\frac{1}{2}$.

3.3. The tight contact structure. We now define the tight contact structure $\xi$ on $M$, using the description of $M$ as a torus bundle. For this we first describe a tight contact structure on $T^{2} \times[0,1]$ with coordinates $(x, y, t)$. Start with a contact structure given by the 1 -form $\alpha=\sin \left(\frac{\pi t}{2}\right) d x+\cos \left(\frac{\pi t}{2}\right) d y$. Perturb the boundary so that $T_{i}=T^{2} \times\{i\}, i=0,1$, are convex with $\# \Gamma_{T_{i}}=2$, and slopes $s\left(\Gamma_{T_{0}}\right)=0, s\left(\Gamma_{T_{1}}\right)=\infty$. Now we identify $T_{0}$ and $T_{1}$ via $A$ to obtain a contact structure on the quotient $M=\left(T^{2} \times[0,1]\right) / \sim$. This is possible since $A\left(\Gamma_{T_{1}}\right)$ is isotopic to $\Gamma_{T_{0}}$ — we may apply Giroux's Flexibility Theorem to ensure that the characteristic foliations agree. 
Proposition 3.2. The contact structure $\xi$ is a virtually overtwisted tight contact structure on $M$.

In fact, $\xi$ is the unique virtually overtwisted tight structure on $M$ (see [22]). The uniqueness is not required in this paper.

Proof. The proof can be found in [22], but we reproduce it here for completeness. We first show the existence of a double cover $\pi: M^{\prime} \rightarrow M$ for which $\pi^{*} \xi$ is overtwisted. If $M=\left(T^{2} \times[0,1]\right) / \sim$, then take two copies $C_{1}$ and $C_{2}$ of $\left(T^{2} \times[0,1], \xi\right)$ and map $T_{1} \subset C_{1}$ to $T_{0} \subset C_{2}$ by $A$ and $T_{1} \subset C_{2}$ to $T_{0} \subset C_{1}$ by $A$. In other words, $M^{\prime}$ is the double cover of $M$ given by $\left(T^{2} \times[0,2]\right) / \sim$, where $\left(A^{2} x, 0\right) \sim(x, 2)$. We may assume $\# \Gamma_{T_{i}}=2$, $i=0,1,2$, and $s\left(\Gamma_{T_{0}}\right)=s\left(\Gamma_{T_{2}}\right)=0, s\left(\Gamma_{T_{1}}\right)=\infty$. If the relative Euler class on $\left(T^{2} \times[0,1], \pi^{*} \xi\right)$ is $e\left(T^{2} \times[0,1], \pi^{*} \xi\right)=(0,1)-(1,0)=(-1,1)$, then $e\left(T^{2} \times[1,2], \pi^{*} \xi\right)=(1,1)$, and $e\left(T^{2} \times[0,2], \pi^{*} \xi\right)=(0,2)$, which is not a possible relative Euler class for a tight contact structure with the given boundary slopes. (See [21] for a discussion of the relative Euler class.) Therefore $\pi^{*} \xi$ is overtwisted. We leave it as an exercise for the reader to explicitly find an overtwisted disk in $T^{2} \times[0,2]$.

We now prove the tightness of $\xi$. To construct $\xi$ on $M$, we started with a tight contact structure $\left.\xi\right|_{T^{2} \times[0,1]}$. If there is an overtwisted disk $D \subset M$, then necessarily $D \cap T_{0} \neq \emptyset$. Below we show how to inductively choose a different torus $T \subset M$ isotopic to $T_{0}$ that does not intersect $D$, and for which $\left.\xi\right|_{M \backslash T}$ is tight. This would contradict the initial assumption of the existence of an overtwisted disk.

Without loss of generality we may assume that $D \pitchfork T_{0}$ and $D \cap T_{0}$ is a disjoint collection of arcs and circles. We first show how to eliminate an outermost arc of intersection. Let $\alpha$ be an outermost arc of $D \cap T_{0}$ on $D$. The arc $\alpha$ then separates off a half-disk $D^{\prime}$ from $D$ that does not intersect $T_{0}$ except along $\alpha$. If we cut open $M$ to obtain $T^{2} \times[0,1]$, then $D^{\prime}$ is attached to either $T_{0}$ or $T_{1}$. We assume the latter (the argument in the other case is similar). Let $N$ be a small closed neighborhood of $T_{1} \cup D^{\prime}$ in $T^{2} \times[0,1]$. The neighborhood $N$ is a toric annulus which, by altering the product structure, we may call $T^{2} \times\left[\frac{1}{2}, 1\right]$. By taking $N$ to be small enough, we can ensure that $T_{1 / 2}=T^{2} \times\left\{\frac{1}{2}\right\}$ has the same (isotopy class of) intersections with $D$ as $D \cap T_{1}$, except for the absence of $\alpha$. Moreover, we may assume that $T_{1 / 2}$ is convex. (For the moment we assume that $\# \Gamma_{T_{1 / 2}}=2$. Below we show how to handle a convex torus with more than two dividing curves.) Now glue $T_{1}$ to $T_{0}$ using the map $A$ to obtain a toric annulus which we denote $T^{2} \times\left[-\frac{1}{2}, \frac{1}{2}\right]$. Note that $(M, \xi)$ can be reconstructed by gluing the two boundary components of $T^{2} \times\left[-\frac{1}{2}, \frac{1}{2}\right]$, and $T_{1 / 2} \subset M$ is a convex torus that has one fewer arc of intersection with $D$ than $D \cap T_{0}$.

Finally, we prove the contact structure $\left.\xi\right|_{T^{2} \times[-1 / 2,1 / 2]}$ is tight. By the classification of tight contact structures on toric annuli [21, 17], any convex torus $T$ in $T^{2} \times[0,1]$ has slope $s(T) \leq 0$. Let $s\left(T_{1 / 2}\right)=-\frac{p}{q}<0$. If $s\left(T_{1 / 2}\right)=$ 0 , then we effectively did not modify the contact structure above. $T^{2} \times$ 
$\left[-\frac{1}{2}, \frac{1}{2}\right]$ is a toric annulus with convex boundary having boundary slopes $s\left(T_{-1 / 2}\right)=\frac{q}{p}$ and $s\left(T_{1 / 2}\right)=-\frac{p}{q}$ that was obtained by gluing $T^{2} \times\left[-\frac{1}{2}, 0\right]$, a toric annulus with (universally) tight contact structure and boundary slopes $\frac{q}{p}$ and 0 , and $T^{2} \times\left[0, \frac{1}{2}\right]$, a toric annulus with (universally) tight contact structure and boundary slopes 0 and $-\frac{p}{q}$. Using the classification of tight contact structures on toric annuli, we claim there is a tight contact structure on $T^{2} \times\left[-\frac{1}{2}, \frac{1}{2}\right]$ with boundary slopes $\frac{q}{p}$ and $-\frac{p}{q}$ that may be split along the convex torus $T_{0}$ with $s\left(T_{0}\right)=0$, so that the pieces are contactomorphic to the contact structures induced on $T^{2} \times\left[-\frac{1}{2}, 0\right]$ and $T^{2} \times\left[0, \frac{1}{2}\right]$. This may easily be seen using Theorem 4.24 in 21] which discusses the gluing of tight contact structures on toric annuli. We have therefore eliminated one outermost arc of intersection from $D$ with a convex torus in $M$ that cuts $M$ into a toric annulus with a tight contact structure. It is not hard to continue this procedure to eliminate other outermost arcs of intersection.

We now eliminate circle components of $D \cap T_{1}$. If $c$ is an innermost circle of $D \cap T_{1}$ on $D$, then $c$ bounds disks $D^{\prime} \subset D$ and $D^{\prime \prime} \subset T_{1}$, and $D^{\prime} \cup D^{\prime \prime}$ bounds a ball $B^{3} \subset T^{2} \times[0,1]$. We may now apply the same argument as above to a small neighborhood $N$ of $T_{1} \cup B$ to eliminate $c$ (and possibly other curves) from $D \cap T_{1}$. At each step of this reduction process, there exists a convex torus $T$ which splits $M$ into a toric annulus with tight contact structure. It is easy to see that by eliminating outermost arcs and innermost circles of $D \cap T$, we can eventually make $D \cap T=\emptyset$, thus proving $\xi$ is tight.

Recall we must still discuss what happens if the new splitting torus $T_{1 / 2}$ constructed above has more than two dividing curves. In this case, Proposition 5.8 in 21] implies there is a contact structure on a neighborhood $T^{2} \times\left[\frac{1}{2}-\varepsilon, \frac{1}{2}+\varepsilon\right]$ of $T_{1 / 2}$ which is $S^{1}$-invariant in the direction given by $s\left(T_{1 / 2}\right)$ and contains a convex $T$ disjoint from $T_{1 / 2}$, with $s(T)=s\left(T_{1 / 2}\right)$ and $\# \Gamma_{T}=2$. If we use $T$ as the new splitting torus, then $\left.\xi\right|_{M \backslash T}$ will be tight (this is just the argument above). But then $\left.\xi\right|_{M \backslash T_{1 / 2}}$ will also be tight since there exists a contact embedding

$$
\left(M \backslash T_{1 / 2},\left.\xi\right|_{M \backslash T_{1 / 2}}\right) \hookrightarrow\left(M \backslash T,\left.\xi\right|_{M \backslash T}\right) .
$$

\subsection{Maximizing the twisting number of a regular fiber.}

Lemma 3.3. There exists a Legendrian knot $F$ in $(M, \xi)$ isotopic to a regular fiber of the Seifert fibered structure with $t(F)=0$.

Lemma 3.3 follows immediately from the following lemma, together with Proposition 3.2.

Lemma 3.4. If $\xi^{\prime}$ is a tight contact structure on $M$ and all Legendrian curves isotopic to a regular fiber have negative twisting number, then $\xi^{\prime}$ is universally tight. 
Proof. To prove $\xi^{\prime}$ is universally tight, we will first show that it can be made transverse to the $S^{1}$-fibers of the Seifert fibration. We then show that this implies that $\left(M, \xi^{\prime}\right)$ is covered by a tight contact structure on the 3 -torus. Since all of the tight contact structures on the 3-torus are universally tight (see [24, 17]), $\xi^{\prime}$ must therefore be universally tight.

Step 1. (Normalization of contact structure $\xi^{\prime}$.) Let $F$ be a Legendrian curve isotopic to a regular fiber with $t(F)=n<0$, which we take to be maximal among Legendrian curves isotopic to a regular fiber. Let $L_{i}$, $i=1,2,3$, be Legendrian curves (simultaneously) isotopic to the singular fibers $F_{i}$ with $t\left(L_{i}\right)=n_{i}<0$, and let $V_{i}$ be a standard convex neighborhood of $F_{i}$; assume also that $t\left(L_{i}\right)$ is maximal among Legendrian curves isotopic to $F_{i}$ with negative twisting number. After making the Legendrian ruling curves on $V_{i}$ vertical (i.e., parallel to the regular $S^{1}$-fibers), take a convex annulus $\mathcal{A}$ with Legendrian boundary for which one component of $\partial \mathcal{A}$ is a ruling curve on $V_{2}$ and the other component is a ruling curve on $V_{3}$. If not all dividing curves on $\mathcal{A}$ connect between $V_{2}$ and $V_{3}$, then the Imbalance Principle (see [21]) gives rise to a bypass along a ruling curve for, say, $V_{2}$. Provided $t\left(L_{2}\right)<-1$, the Twist Number Lemma (see [21]) implies the existence of a Legendrian curve isotopic to $L_{2}$ with larger twisting number. Therefore, we conclude that either $n_{2}=n_{3}<-1$ and $\mathcal{A}$ has no $\partial$-parallel dividing curves, or $n_{2}=n_{3}=-1$.

Assume $n_{2}=n_{3} \leq-1$ and $\mathcal{A}$ has no $\partial$-parallel dividing curves. If $N(\mathcal{A})$ is a convex neighborhood of $\mathcal{A}$, then $M^{\prime}=V_{2} \cup V_{3} \cup N(\mathcal{A})$ will have a piecewise smooth convex torus boundary. Rounding the corners in the standard way (see [21]), $M^{\prime}$ will be a convex torus with boundary slope

$$
\frac{-n_{2}}{4 n_{2}+1}+\frac{-n_{2}}{4 n_{2}+1}+\frac{-1}{4 n_{2}+1}=-\frac{2 n_{2}+1}{4 n_{2}+1},
$$

measured using the identification $\partial\left(M \backslash V_{1}\right) \simeq \mathbf{R}^{2} / \mathbf{Z}^{2}$. Now $M^{\prime \prime}=\overline{M \backslash M^{\prime}}$ is a solid torus with convex boundary with slope $-\frac{2 n_{2}+1}{4 n_{2}+1}$, measured using $\partial\left(M \backslash V_{1}\right)$, which is equivalent to slope $\frac{1}{2 n_{2}+1}$ measured using $\partial V_{1}$. This implies that the contact structure on $M^{\prime \prime}$ is the unique contact structure on the standard neighborhood of the Legendrian knot $L_{1}$ with $n_{1}=2 n_{2}+1$.

Now assume $n_{2}=n_{3}=-1$ and $\mathcal{A}$ has $\partial$-parallel dividing curves. Then we use the corresponding bypasses to thicken $V_{2}, V_{3}$ to $V_{2}^{\prime}, V_{3}^{\prime}$ so that the boundary slopes (measured on $\left.-\partial\left(M \backslash V_{i}\right)\right)$ are $-\frac{1}{2},-\frac{1}{2}$ or $-1,-1$ and the dividing curves on $\mathcal{A}$ between $V_{2}^{\prime}$ and $V_{3}^{\prime}$ do not have $\partial$-parallel curves. The former case gives an overtwisted contact structure and the latter yields a Legendrian curve isotopic to a regular fiber with $t=0$.

Summarizing, $\xi^{\prime}$ has been normalized so that $V_{2}$ and $V_{3}$ are standard neighborhoods of Legendrian curves with twisting number $n_{2}=n_{3}, \mathcal{A}$ has no $\partial$-parallel dividing curves, and $V_{1}=M \backslash\left(V_{2} \cup V_{3} \cup N(\mathcal{A})\right)$ is a standard neighborhood of a Legendrian curve with twisting number $n_{1}=2 n_{2}+1$. 
Step 2. (Making $\xi^{\prime}$ transverse to the fibers.) Initially $K=\partial V_{2} \cup \partial V_{3} \cup \mathcal{A}$ has Legendrian rulings by vertical curves. We perturb $K$ slightly so that the characteristic foliation becomes nonsingular Morse-Smale, and $V_{2} \cap \mathcal{A}$ and $V_{3} \cap \mathcal{A}$ become transverse to $\xi^{\prime}$. Since $\partial V_{2}, \partial V_{3}$, and $\mathcal{A}$ are all convex in standard form, it is possible to perturb $K$ along the Legendrian divides as in [22] to accomplish this. Now, it is a question of isotoping $\xi^{\prime}$ so that $\xi^{\prime}$ is transverse to the fibers on each $V_{i}$. Let us consider $V_{2}$, for example, and use the identification $\partial V_{2} \simeq \mathbf{R}^{2} / \mathbf{Z}^{2}$ to measure slope. The regular fibers of the Seifert fibration have slope -4 , and the nonsingular Morse-Smale characteristic foliation has dividing curves of slope $-\frac{1}{n_{2}}$. Since $-4<-\frac{1}{n_{2}}$, it is clearly possible to extend $\left.\xi^{\prime}\right|_{\partial V_{2}}$ so that the contact structure is transverse to the Seifert fibers. Moreover, this extension is contact isotopic to $\xi^{\prime}$ rel $\partial V_{2}$. In this way, we isotop $\xi^{\prime}$ so that $\xi^{\prime}$ is transverse to the $S^{1}$-fibers of $M$.

Step 3. (Pulling back to $T^{3}$.) Since the monodromy of $M$ as a torus bundle has order four, there exists a 4-fold cover $\pi: T^{3}=T^{2} \times S^{1} \rightarrow M$ with the property that $\{p t\} \times S^{1} \subset T^{3}$ are lifts of fibers of $M$. The pullback $\pi^{*} \xi^{\prime}$ is therefore transverse to the fibers $\{p t\} \times S^{1}$ of $T^{3}$.

Step 4. (Universal tightness.) A tight contact structure on $T^{3}$ is universally tight by the classification results of Kanda [24] and Giroux [17]. Therefore, in order to show $\xi^{\prime}$ is universally tight, it suffices to show that $\left(T^{3}, \pi^{*} \xi^{\prime}\right)$ is tight.

We prove the well-known fact that a contact structure $\zeta$ on $T^{2} \times S^{1}$ which is transverse to the fibers must be tight. (The proof extends easily to any circle bundle.) Let $\pi_{1}, \pi_{2}$ be the projections of $T^{2} \times D^{2}$ to $T^{2}$ and $D^{2}$ respectively. If $\omega_{1}, \omega_{2}$ are area forms on $T^{2}$ and $D^{2}$, then $\omega=\pi_{1}^{*} \omega_{1}+\pi_{2}^{*} \omega_{2}$ is a symplectic form on $T^{2} \times D^{2}$ that restricts to a symplectic form on $\zeta$. The contact structure $\zeta$ is symplectically fillable and therefore tight.

3.5. Twisting number increase for singular fibers. We need one more result before the proof of Theorem 1.1.

Proposition 3.5. There is a Legendrian knot $L$ in $(M, \xi)$ isotopic to one of the singular fibers $F_{2}$ or $F_{3}$ with $t(L)=0$.

Proof. Let $F$ be a Legendrian knot isotopic to a regular fiber with $t(F)=0$ as in Lemma 3.3. Let $V_{i}^{\prime}, i=1,2,3$, be disjoint solid tori isotopic to tubular neighborhoods of $F_{i}$, for which $\partial V_{i}^{\prime}$ contains a contact-isotopic copy of $F$. By perturbing $\partial V_{i}^{\prime}$ we may assume $V_{i}^{\prime}$ is convex with vertical (i.e., parallel to the regular fibers) dividing curves, and, furthermore, we may assume that $\# \Gamma_{\partial V_{i}^{\prime}}=2$, after possibly taking a smaller solid torus. In order to increase the twisting number of a Legendrian curve, we need to find a bypass. We will find a bypass along, say, $V_{3}^{\prime}$ by patching together meridional disks of $V_{1}^{\prime}$ and $V_{2}^{\prime}$ to obtain a punctured torus $T$ and showing the existence of a $\partial$-parallel dividing curve on $T$. 
Step 1. (Normalizing $\xi$ on the complement.) Let us first normalize the tight contact structure on $\Sigma_{0} \times S^{1}=M \backslash\left(V_{1}^{\prime} \cup V_{2}^{\prime} \cup V_{3}^{\prime}\right)$.

Lemma 3.6. The contact structure on $\Sigma_{0} \times S^{1}$ is contactomorphic to a $[0,1]$-invariant tight contact structure on $T^{2} \times[0,1]$ with convex boundary, $\# \Gamma_{T^{2} \times\{i\}}=2, i=1,2$, and slopes $s\left(T^{2} \times\{i\}\right)=\infty$, (i.e., the tight contact structure induced on $T^{2} \times[0,1]$, thought of as a neighborhood of a convex torus in standard form) and a standard (open) neighborhood of a vertical (i.e., isotopic to $\{p t\} \times S^{1} \subset T^{2}$ ) Legendrian curve with 0 twisting removed.

Sketch of proof. This lemma is proved in [15] ( cf. [22]), but we sketch the basic idea. Since $\xi$ is tight on $M$, no dividing curve on $\Sigma_{0}$ can be $\partial$-parallel. This leaves us with two possibilities (depending on signs) for the dividing curves on $\Sigma_{0}$, modulo spiraling. Cutting $\Sigma_{0} \times S^{1}$ open along $\Sigma_{0}$, we obtain a tight contact structure on a genus two handlebody with a fixed set of dividing curves. In addition the dividing curves are such that one can use techniques in [24] to show there is a unique tight contact structure on the handlebody. From this we can conclude that there is a unique tight contact structure on $\Sigma_{0} \times S^{1}$ with the given dividing curve data. Now, since the contact structure described in the lemma also has this dividing curve data, our contact structure must be contactomorphic to it.

Step 2. (Patching meridional disks.) If we measure slopes of $\partial V_{i}^{\prime}, i=1,2,3$, using the identification $\partial V_{i} \simeq \mathbf{R}^{2} / \mathbf{Z}^{2}$, then the slopes are 2, -4 and -4 , respectively. After making the ruling curves on $\partial V_{i}^{\prime}$ meridional, a convex meridional disk $D_{i}$ for $V_{i}^{\prime}$ will have, respectively, $t b\left(\partial D_{i}\right)=-2,-4,-4$, and also 2, 4 and 4 dividing curves. We would like to patch copies of the meridional disks together to create a convex surface and moreover relate information about the dividing curves on this patched-together surface to the dividing curves on the meridional disks.

We view the $T^{2} \times I$ (minus $S^{1} \times D^{2}$ ) from Lemma 3.6 as the region between $\partial V_{1}^{\prime}$ and $\partial V_{2}^{\prime}$ (minus $V_{3}^{\prime}$ ). Write $T_{t}=T^{2} \times\{t\}, t \in[0,1]$, as before. Assume $T_{0}=\partial V_{2}^{\prime}$ and $T_{1}=-\partial V_{1}^{\prime}$. Since $\xi$ is $I$-invariant, we have (for example) a 1-parameter family of positive regions $\left(T_{t}\right)_{+}=\left(T^{2}\right)_{+} \times\{t\}$. We may then isotop $T_{i}, i=0,1$, away from $\left(T_{i}\right)_{+}$(i.e., on $\left.\left(T_{i}\right)_{-}\right)$to arrange the slopes of the Legendrian ruling curves so that the meridional disk $D_{i}$ in $V_{i}^{\prime}$ has Legendrian boundary. Now, take one copy of $D_{2}$ and two copies $D_{11}, D_{12}$ of $D_{1}$, and arrange them so that $D_{2} \cap\left(T_{0}\right)_{+}=\delta \times\{0\}$ and $\left(D_{11} \cup D_{12}\right) \cap\left(T_{1}\right)_{+}=$ $\delta \times\{1\}$, where $\delta$ is a union of Legendrian arcs on $\left(T^{2}\right)_{+}$with endpoints on opposite ends of $\partial\left(T^{2}\right)_{+}$. Let $T=D_{11} \cup D_{12} \cup D_{2} \cup(\delta \times[0,1])$, which is a torus with an open disk removed. See Figure 2. After smoothing the corners using the 'elliptic monodromy lemma' or the 'pivot lemma' of [14, 11], $T$ will have smooth Legendrian boundary. Since $\partial T \subset \partial\left(\left(T^{2}\right)_{-} \times[0,1]\right)$, we shall think of $T$ as having its boundary on $\partial V_{3}^{\prime}$.

Step 3. (Combinatorics of $D_{1}, D_{2}$ and $D_{3}$.) Since $D_{1}$ has two dividing curves, it either has two positive regions and one negative region, or one 


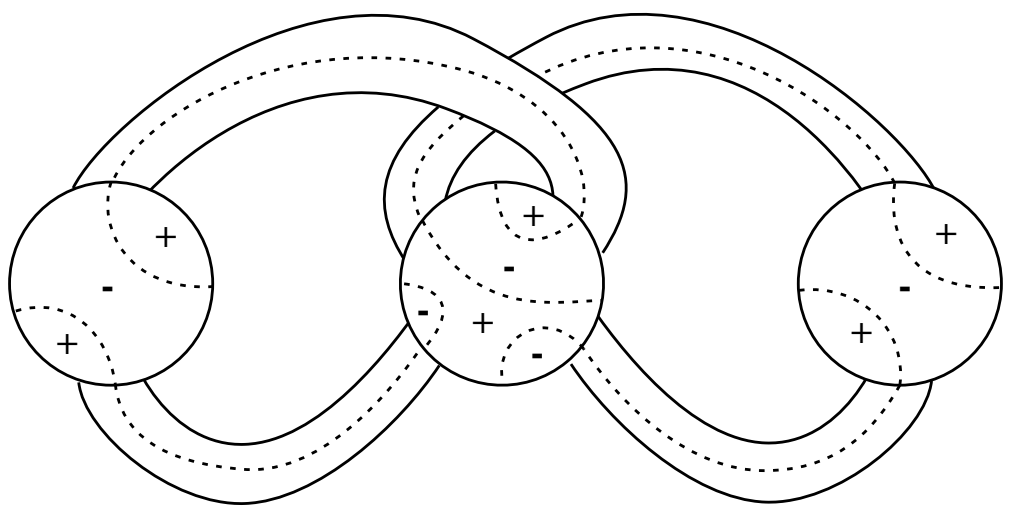

FigurE 2. The punctured torus $T$ with dividing curves (dashed lines).

positive and two negative regions. We assume the former - the argument for the latter is identical.

The rotation number $r\left(\partial D_{i}\right), i=2,3$, satisfies the formula $r\left(\partial D_{i}\right)=$ $\chi\left(\left(D_{i}\right)_{+}\right)-\chi\left(\left(D_{i}\right)_{-}\right)$in [25]. Therefore, $r\left(\partial D_{i}\right)$ can attain values $-3,-1,1,3$.

Step 3A. Assume that at least one of $D_{2}$ or $D_{3}$ (say $D_{2}$, after possible relabeling) satisfies $r\left(\partial D_{i}\right)>-3$. We first show that $D_{2}$, after possibly isotoping rel $\partial D_{2}$, will have a positive $\partial$-parallel region. If $r\left(\partial D_{2}\right)=3$ or 1 , there is no problem. If $r\left(\partial D_{2}\right)=-1$, the dividing curves on $D_{2}$ may be either of the two types shown in Figure 3. If we have a configuration shown
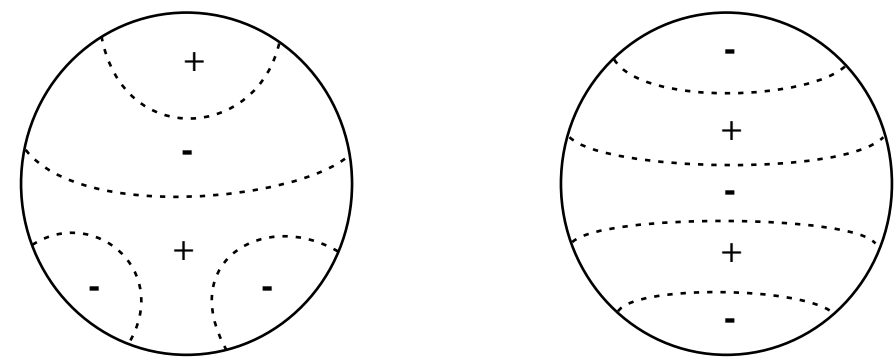

Figure 3. Possible dividing curves on $D_{2}$.

on the right-hand side of Figure 3, then we may isotop $D_{2}$ rel $\partial D_{2}$ so that the dividing curves on $D_{2}$ are as shown on the left-hand side of Figure 3 . This follows from the classification of tight contact structures on solid tori in 21] or 17].

If $r\left(\partial D_{2}\right)=-1$, then we take the dividing curves on $D_{2}$ to be as shown on the left-hand side of Figure 3. The dividing curves on $T$ will then be as shown in Figure 2. Note we have a $\partial$-parallel component and hence a bypass along $\partial T$. The cases $r\left(\partial D_{2}\right)=1,3$ are similar, by observing that any positive $\partial$-parallel component of $D_{2}$ must necessarily be connected to a 
positive $\partial$-parallel component on one of the copies of $D_{1}$, yielding a $\partial$-parallel component on $T$.

The slope of $D_{1}$ on $\partial\left(M \backslash V_{1}^{\prime}\right)$ is $-\frac{1}{2}$ and the slope of $D_{2}$ on $\partial\left(M \backslash V_{2}^{\prime}\right)$ is $\frac{1}{4}$. This implies that the slope of $T$ on $-\partial\left(M \backslash V_{3}^{\prime}\right)$ is $-\frac{1}{4}$. We therefore have a bypass on $\partial V_{3}^{\prime}$ attached along a ruling curve of slope $-\frac{1}{4}$ (as measured from $\left.\Sigma_{0} \times S^{1}\right)$. Using this bypass, we may thicken $V_{3}^{\prime}$ to $V_{3}^{\prime \prime}$ with standard convex boundary having boundary slope 0 . Thus, when measured from the product structure $D^{2} \times S^{1}$ on $V_{3}^{\prime \prime}$, the slope is $\infty$, showing that $V_{3}^{\prime \prime}$ is the standard neighborhood of a Legendrian curve $L$ isotopic to $F_{3}$ with twist number 0 .

Step 3B. We are left with the case where $r\left(\partial D_{2}\right)=r\left(\partial D_{3}\right)=-3$. Now the dividing curves on the punctured torus $T$ constructed from $D_{2}$ and two copies of $D_{1}$ will be as in Figure 1 . Capping $T$ off with $D_{3}$, we obtain a

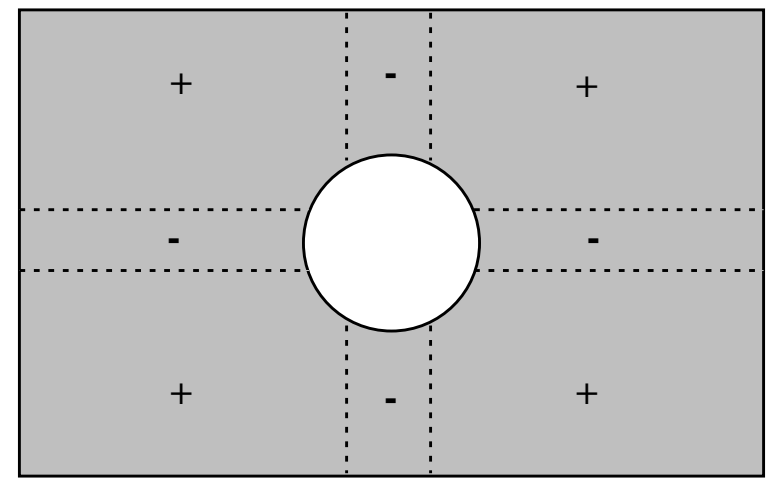

Figure 4. The punctured torus $T$ (shaded).

closed contractible dividing curve on the torus $T \cup D_{3}$ which contradicts tightness.

This completes the proof of Proposition 3.5.

3.6. Proof of Theorem 1.1 for the Seifert fibered space with invariants $\left(-\frac{1}{2}, \frac{1}{4}, \frac{1}{4}\right)$. We are now ready to prove our main theorem.

Proof. From Proposition 3.2 we have a tight contact structure $\xi$ on $M$. Now assume that $\xi$ is weakly symplectically semi-fillable. From Proposition 3.5 we have a Legendrian knot $L$ in $(M, \xi)$ that is isotopic to, say, $F_{3}$ with twist number 0 . We may assume that the neighborhood $V_{3}$ was chosen so that $L=F_{3}$. As discussed above, if we perform Legendrian surgery on $L$, we remove a small neighborhood of $L$ (take this neighborhood to lie in $V_{3}$ ) and re-glue it by $\left(\begin{array}{cc}1 & 0 \\ -1 & 1\end{array}\right)$. We easily see this has the same effect as changing the $A_{3}$ to

$$
\left(\begin{array}{cc}
3 & 1 \\
-1 & 0
\end{array}\right)=\left(\begin{array}{cc}
4 & 1 \\
-1 & 0
\end{array}\right)\left(\begin{array}{cc}
1 & 0 \\
-1 & 1
\end{array}\right)
$$


Thus, after Legendrian surgery we obtain a weakly symplectically semifillable contact structure on $M^{\prime}$, the Seifert fibered space over $S^{2}$ with Seifert invariants $\left(-\frac{1}{2}, \frac{1}{3}, \frac{1}{4}\right)$. This contradicts Lisca's Theorem (Theorem 2.2), thus proving $\xi$ is not weakly symplectically semi-fillable.

3.7. Modifications for the Seifert fibered space with invariants $\left(-\frac{2}{3}, \frac{1}{3}, \frac{1}{3}\right)$. The steps are almost identical for the Seifert fibered space $M_{2}$ over $S^{1}$ with 3 singular fibers and invariants $\left(-\frac{2}{3}, \frac{1}{3}, \frac{1}{3}\right)$. The manifold $M_{2}$ has a presentation as a torus bundle over $S^{1}$ with monodromy $A=$ $\left(\begin{array}{cc}0 & 1 \\ -1 & -1\end{array}\right)$. By the classification in [22], there exist two virtually overtwisted tight contact structures on $M_{2}$ which are nonisotopic but isomorphic. Let $\xi$ be one such contact structure. As before, we first find a Legendrian knot $F$ isotopic to a regular fiber with $t(F)=0$. We then find a Legendrian curve $L$ isotopic to the $-\frac{2}{3}$-fiber $F_{1}$ with $t(L)$ large enough to perform a Legendrian surgery which modifies the Seifert invariants as follows:

$$
\left(-\frac{2}{3}, \frac{1}{3}, \frac{1}{3}\right) \rightsquigarrow\left(-\frac{1}{2}, \frac{1}{3}, \frac{1}{3}\right) \text {. }
$$

This again gives a contradiction of Theorem 2.2. The existence of such an $L$ is proved by patching together meridional disks as in Proposition 3.5 the only difference is that in one case we need to apply a 'thinning before thickening' argument that is used in 15.

\section{Further Questions}

The obvious question raised in this paper is

Question 1. Are the contact structures on the Seifert fibered spaces over $S^{2}$ with Seifert invariants $\left(-\frac{1}{2}, \frac{1}{3}, \frac{1}{3}\right)$ or $\left(-\frac{1}{2}, \frac{1}{3}, \frac{1}{4}\right)$ constructed above tight?

We conjecture that these contact structures are tight. If the conjecture is true, we would have an example of a manifold that supported a tight contact structure but no symplectically fillable contact structures. Since this contact structure is constructed from a tight contact structure by Legendrian surgery, we are led to ask the following:

Question 2. Is Legendrian surgery category-preserving for tight structures on closed 3-manifolds?

If not, are there conditions which are sufficient to guarantee that Legendrian surgery on the contact structure yields a tight contact structure? For example,

Question 3. Does Legendrian surgery on a universally tight contact structure produce a tight contact structure?

Recall our tight but not symplectically fillable contact structure is virtually overtwisted. All other potential tight but not symplectically fillable contact structures known to the authors are also virtually overtwisted. (There are several candidates mentioned in [22].) So we ask 
Question 4. Are all universally tight contact structures symplectically semifillable?

Acknowledgements. We thank Yasha Eliashberg for informing us that Leg-

endrian surgery preserves weakly fillable contact structures.

\section{REFERENCES}

[1] B. Aebisher, et. al., Symplectic Geometry, Progress in Math. 124, Birkhäuser, Basel, Boston and Berlin, 1994.

[2] D. Bennequin, Entrelacements et équations de Pfaff, Astérisque 107-108 (1983), 87-161.

[3] V. Colin, Chirurgies d'indice un et isotopies de sphères dans les variétés de contact tendues, C. R. Acad. Sci. Paris Sr. I Math. 324 (1997), 659-663.

[4] V. Colin, Recollement de variétés de contact tendues, Bull. Soc. Math. France 127 (1999), 43-69.

[5] V. Colin, Chirurgie de Dehn admissible dans une variété de contact tendue, preprint 2000.

[6] Y. Eliashberg, Classification of overtwisted contact structures on 3-manifolds, Invent. math. 98 (1989), 623-637.

[7] Y. Eliashberg, Topological characterization of Stein manifolds of dimension > 2, Int. J. of Math. 1 (1990), 29-46.

[8] Y. Eliashberg, Filling by holomorphic discs and its applications, Geometry of lowdimensional manifolds, Vol. II (Ed. Donaldson and Thomas), Cambridge, 1990.

[9] Y. Eliashberg, Contact 3-manifolds twenty years since J. Martinet's work, Ann. Inst. Fourier 42 (1992), 165-192.

[10] Y. Eliashberg, Unique holomorphically fillable contact structure on the 3-torus, Internat. Math. Res. Notices 2 (1996), 77-82.

[11] Y. Eliashberg and M. Fraser, Classification of topologically trivial Legendrian knots, in Geometry, topology, and dynamics (Montreal, PQ, 1995), 17-51, CRM Proc. Lecture Notes 15, Amer. Math. Soc., Providence, RI, 1998.

[12] Y. Eliashberg and W. Thurston, Confoliations, University Lecture Series 13, Amer. Math. Soc., Providence, 1998.

[13] J. Etnyre, Symplectic convexity in low-dimensional topology, Top. Appl. 88 (1998), $3-25$.

[14] J. Etnyre, Contact structures on lens spaces, to appear in Commun. in Contemp. Math.

[15] J. Etnyre and K. Honda, On the non-existence of tight contact structures, preprint 1999.

[16] E. Giroux, Convexité en topologie de contact, Comment. Math. Helvetici 66 (1991), 637-677.

[17] E. Giroux, Structures de contact en dimension trois et bifurcations des feuilletages de surfaces, Invent math. 141 (2000), 615-689.

[18] R. Gompf, Handlebody construction of Stein surfaces, Annals of Math. 148 (1998), 619-693.

[19] R. Gompf and A. Stipsicz, 4-manifolds and Kirby calculus, Graduate Studies in Mathematics, 20, American Mathematical Society, Providence, RI, 1999.

[20] M. Gromov, Pseudo-holomorphic curves in symplectic manifolds, Invent. math. 82 (1985), 307-347.

[21] K. Honda, On the classification of tight contact structures I, Geom. Topol. 4 (2000), 309-368. 
[22] K. Honda, On the classification of tight contact structures II, to appear in J. Diff. Geom.

[23] K. Honda, Gluing tight contact structures, preprint 2000.

[24] Y. Kanda, The classification of tight contact structures on the 3-torus, Comm. in Anal. and Geom. 5 (1997), 413-438.

[25] Y. Kanda, On the Thurston-Bennequin invariant of Legendrian knots and non exactness of Bennequin's inequality, Invent. math. 133 (1998), 227-242.

[26] P. Kronheimer and T. Mrowka, Monopoles and contact structures, Invent. math. 130 (1997), 209-255.

[27] P. Lisca, On symplectic fillings of 3-manifolds, Proceedings of 6th Gökova GeometryTopology Conference. Turkish J. Math. 23 (1999), 151-159.

[28] P. Lisca and G. Matić, Stein 4-manifolds with boundary and contact structures, Top. Appl. 88 (1998), 55-66.

[29] S. Makar-Limanov, Morse surgeries of index 0 on tight manifolds, preprint 1997.

[30] D. McDuff and D. Salamon, Introduction to symplectic topology, Oxford University Press, 1995.

[31] H. Ohta and K. Ono, Simple singularities and topology of symplectically filling 4manifold, Comment. Math. Helv. 74 (1999), 575-590.

[32] L. Rudolph, An obstruction to sliceness via contact geometry and "classical" gauge theory, Invent. math. 119 (1995), 155-163.

[33] A. Weinstein, Contact surgery and symplectic handlebodies, Hokkiado Math. Journal 20 (1991), 241-251.

Stanford University, Stanford, CA 94305

E-mail address: etnyre@math.stanford.edu

$U R L:$ http://math. stanford.edu/ ${ }^{\sim}$ etnyre

University of Georgia, Athens, GA 30602

E-mail address: honda@math.uga.edu

$U R L$ : http://www . math.uga.edu/ honda 